\title{
ON APPROXIMATION TO FUNCTIONS SATISFYING A GENERALIZED CONTINUITY CONDITION
}

\author{
BY \\ H. MARGARET ELLIOTT( $\left.{ }^{1}\right)$
}

1. Introduction. Let $C$ consist of a finite number of mutually exterior rectifiable Jordan curves in the complex $z$-plane; denote the sum of their closed interiors by $\bar{C}$. Let $u(z)$ be harmonic in the interior points of $\bar{C}$ and continuous on $\bar{C}$. The purpose of the present paper is to study the relation between the modulus of continuity of $u(z)$ on $C$ and approximation to $u(z)$ on $\bar{C}$ by harmonic polynomials when degree of approximation is measured (i) in the sense of Tchebycheff and (ii) in the sense of least $p$ th powers. The corresponding problem for analytic functions is also treated. The special case that $u(z)$ satisfies a Lipschitz condition of order $\alpha, 0<\alpha \leqq 1$, has been extensively studied (for example [11]) $\left({ }^{2}\right)$.

Let $w=\phi(z)$ map the exterior of $C$ conformally (not necessarily uniformly) onto $|w|>1$ so that the points at infinity in the two planes correspond. Denote by $C_{R}$ the locus $|\phi(z)|=R, R>1$, which lies in the exterior of $C$. Our study of approximation to functions by sequences of polynomials is divided into Problems $\alpha$ and $\beta$. In Problem $\alpha$ we investigate the relation between continuity properties on $C$ of the function and degree of convergence on $\bar{C}$ of approximating polynomials; in Problem $\beta$ we investigate the relation between continuity properties on $C_{R}$ of the function and degree of convergence on $\bar{C}$ of the approximating polynomials. For each of these problems, results obtained are of two types: direct theorems and indirect theorems. In a direct theorem given certain continuity properties of a function, we establish the existence and degree of convergence of a sequence of approximating polynomials; in an indirect theorem given a sequence of polynomials converging to a function with a certain degree, we establish continuity properties of the function.

We shall understand by a harmonic function or harmonic polynomial a real harmonic function or polynomial. The letter $s$ will denote arc-length measured along the curve in question; $\partial^{k} u(z) / \partial s^{k}$ will indicate the $k$ th derivative of $u(z)$ with respect to arc-length $s$. For any function $F(z), F^{(0)}(z) \equiv F(z)$. The letter $k$ will be reserved for positive integers and zero. The letters $L$ and $M$ with or without subscripts denote positive constants which may vary from one theorem and its proof to another and may depend on the point sets involved,

Presented to the Society, October 30, 1948; received by the editors August 8, 1950.

(1) A portion of this material is from my doctorate thesis written at Radcliffe College under the direction of Professor J. L. Walsh; part of it was obtained while I held the Vassie James Hill A.A.U.W. Fellowship.

(2) Numbers in brackets refer to the bibliography at the end of the paper. 
but which are independent of $w, z, \delta, m$, and $n$.

We use throughout the Lebesgue integral. Measure on a rectifiable Jordan curve is defined in terms of arc-length $s$.

2. Properties of the modulus of continuity. We study in this section certain properties of the modulus of continuity which will be needed in the remainder of the paper. Let $E$ be a closed bounded set in the $z$-plane which consists of the mutually disjoint Jordan arcs, Jordan curves, and closed Jordan regions $E_{j}, j=1, \cdots, \mu$. Let $F(z)$ be a real or complex function which is continuous on each $E_{j}$. The modulus of continuity $\omega_{j}(\delta)$ of $F(z)$ on $E_{j}$ shall be defined as the maximum of $\left|F\left(z_{1}\right)-F\left(z_{2}\right)\right|$ for all $z_{1}$ and $z_{2}$ in $E_{j}$ such that $\left|z_{1}-z_{2}\right| \leqq \delta$. The modulus of continuity $\omega(\delta)$ of $F(z)$ on $E$ shall be defined as the maximum of $\omega_{j}(\delta), 1 \leqq j \leqq \mu$. If $F(z)$ satisfies a Lipschitz condition of order $\alpha$ on $E$, then clearly $\omega(\delta) \leqq L \delta^{\alpha}$. It follows from the definition of $\omega(\delta)$ that $\omega(\delta)$ is a nondecreasing function of $\delta$ and that $\lim _{\delta \rightarrow 0} \omega(\delta)=0$.

Moreover, if $\omega\left(\delta_{1}\right)=0$ for some $\delta_{1} \neq 0$, then $F(z) \equiv L_{j}$ for $z$ in $E_{j}$, and hence $\omega(\delta) \equiv 0$. For let $z_{0}$ be any point of $E_{j} ; F(z)$ is constant on the set of points $\zeta$ which belong to $E_{j}$ and are such that $\left|\zeta-z_{0}\right| \leqq \delta_{1}$. By covering $E_{j}$ with a finite number of suitably chosen overlapping circles, we obtain that $F(z)$ is identically constant in $E_{j}$.

THEOREM 2.1. If the $E_{j}$ are convex and $\lambda>0$, then $\omega(\lambda \delta) \leqq(\lambda+1) \omega(\delta)$.

The method used by de la Vallée Poussin [4, p. 8] to prove the corresponding theorem for real functions of a real variable yields $\omega_{j}(\lambda \delta)$ $\leqq(\lambda+1) \omega_{j}(\delta)$; hence $\omega_{j}(\lambda \delta) \leqq(\lambda+1) \omega(\delta)$, and $\omega(\lambda \delta) \leqq(\lambda+1) \omega(\delta)$.

THEOREM 2.2. Let the $E_{j}$ consist of a finite number of mutually disjoint rectifiable Jordan arcs and curves. If for every arc of each $E_{j}$, the ratio of the length of that arc to the length of its corresponding chord is less than a constant $A$, and if $\lambda>0$, then $\omega(\lambda \delta) \leqq(A \lambda+1) \omega(\delta)$.

Suppose $F(z)=F_{j}^{*}(s)$, $z$ on $E_{j}$. Denote by $\omega_{j}^{*}(\delta)$ the modulus of continuity of $F_{j}^{*}(s)$ on $0 \leqq s \leqq \sigma_{j}, \sigma_{j}$ the length of $E_{j}$. By the use of Theorem 2.1 we obtain

$$
\omega_{j}(\lambda \delta) \leqq \omega_{j}^{*}(A \lambda \delta) \leqq(A \lambda+1) \omega_{j}^{*}(\delta) \leqq(A \lambda+1) \omega_{j}(\delta) \leqq(A \lambda+1) \omega(\delta),
$$

and the theorem follows.

THEOREM 2.3. Let the sets $E_{j}$ consist of the respective closed interiors of the mutually exterior Jordan curves $C_{j}$; let $z=\chi_{j}(w)$ map $|w|<1$ one-to-one and conformally onto the interior of $C_{j}$. If for all $w_{1}$ and $w_{2}$ on $|w|=1$ we have

$$
\begin{aligned}
L_{1}\left|w_{1}-w_{2}\right| \leqq\left|\chi_{j}\left(w_{1}\right)-\chi_{j}\left(w_{2}\right)\right| \leqq L_{2}\left|w_{1}-w_{2}\right|, & \\
& j=1, \cdots, \mu,
\end{aligned}
$$

and if $\lambda>0$, then $\omega(\lambda \delta) \leqq\left(L_{2} \lambda / L_{1}+1\right) \omega(\delta)$. 
Under the hypothesis on the $C_{j}$, it is known (see for example [7, Theorem 1.2.7]) that inequality (2.1) is valid for $\left|w_{1}\right| \leqq 1,\left|w_{2}\right| \leqq 1$. A slight modification of the method used to prove Theorem 2.2 then yields Theorem 2.3.

THEOREM 2.4. If the sets $E_{j}$ satisfy the hypothesis of any of Theorems 2.1, 2.2 , or 2.3 , and if $\omega(\delta) \not \equiv 0$, then there exists a constant $L$ depending only on $E$ such that

$$
\delta \leqq L \omega(\delta), \quad \text { for } 0 \leqq \delta \leqq 1 .
$$

The hypothesis implies $\omega(1) \neq 0$. If $\delta>0$, it follows from the appropriate one of Theorems $2.1,2.2,2.3$ that $\omega(1) \leqq\left(L_{3} / \delta+1\right) \omega(\delta)$. Hence $\delta$ $\leqq\left[\left(L_{3}+1\right) / \omega(1)\right] \omega(\delta)$ for $0 \leqq \delta \leqq 1$.

We shall in the remainder of this paper frequently use the notation $\omega(\delta)$ for a majorizing function of the modulus of continuity rather than for the actual modulus of continuity. When $\omega(\delta)$ does denote the modulus of continuity, this fact will always be stated. If $\omega_{1}(\delta)$ denotes a majorizing function of the modulus of continuity $\omega(\delta)$, then Theorem 2.4 is clearly valid when $\omega(\delta)$ is replaced by $\omega_{1}(\delta)$ in inequality (2.2).

By the use of simple algebraic inequalities the following result is easily proved:

TheOREM 2.5. Let $F(z)$ and $G(z)$ be two real or complex functions continuous on $E$, where $E$ is composed of a finite number of mutually exterior Jordan arcs, Jordan curves, and closed Jordan regions. Suppose the respective moduli of continuity of $F(z)$ and $G(z)$ on $E$ are not greater than $\omega(\delta)$. Then (i) the modulus of continuity of $F(z) \cdot G(z)$ on $E$ is less than $M_{1} \omega(\delta)$; (ii) if $|F(z)|$ is bounded from zero on $E$, the modulus of continuity of $1 / F(z)$ on $E$ is less than $M_{2} \omega(\delta)$; (iii) if $F(z) \geqq L>0$ on $E$, the modulus of continuity of $[F(z)]^{1 / 2}$ on $E$ is less than $M_{3} \omega(\delta)$.

We study next the effect of certain conformal mappings on the modulus of continuity. Let $C$ be a rectifiable Jordan curve in the $z$-plane; let $z=\chi(w)$, where $w=r e^{i \theta}$, map $|w|<1$ one-to-one and conformally onto the interior of $C$. Set $\chi\left(e^{i \theta}\right)=\zeta(\theta)+i \eta(\theta)$.

THEOREM 2.6. Let $C$ be a rectifiable Jordan curve such that for every arc of $C$ the ratio of the length of that arc to the length of its corresponding chord is less than a constant $A$; suppose there exist constants $L_{1}$ and $L_{2}$ such that $L_{1}|\delta|$ $\leqq\left|\chi\left(e^{i(\theta+\delta)}\right)-\chi\left(e^{i \theta}\right)\right| \leqq L_{2}|\delta|$. Let $F(z)$ be continuous on $C$, and suppose $\partial^{k} F(z) / \partial s^{k}$ is continuous on $C$ with modulus of continuity $\omega(\delta)$. If $k \geqq 1$, suppose $\zeta^{(k)}(\theta), \eta^{(k)}(\theta)$ exist and are continuous on $0 \leqq \theta \leqq 2 \pi$ with moduli of continuity not greater than $L_{3} \omega(\delta)$. Set $F\left[\chi\left(e^{i \theta}\right)\right]=h(\theta)$. Then the modulus of continuity of $h^{(k)}(\theta)$ on $0 \leqq \theta \leqq 2 \pi$ is less than $M \omega(\delta)$.

Except in the trivial case that $F(z)$ is identically constant, we have $\omega(\delta)$ 
$\not \equiv 0$. For $k=0$, Theorem 2.6 is easily established by the method used to prove Theorem 2.2.

For $k \geqq 1$, the hypothesis on $C$ and Theorem 2.4 yield for $\left|\theta_{1}-\theta_{2}\right| \leqq \delta$ :

$$
\left|\frac{\partial^{j} F\left(z_{1}\right)}{\partial s^{j}}-\frac{\partial^{j} F\left(z_{2}\right)}{\partial s^{j}}\right| \leqq \int_{z_{1}}^{z_{2}}\left|\frac{\partial^{i+1} F}{\partial s^{j+1}}\right| d s \leqq M_{1}\left|\theta_{1}-\theta_{2}\right| \leqq M_{2} \omega(\delta),
$$

$$
\left|\frac{\partial^{k} F\left(z_{1}\right)}{\partial s^{k}}-\frac{\partial^{k} F\left(z_{2}\right)}{\partial s^{k}}\right| \leqq \omega\left(L_{2} \delta\right) \leqq M_{3} \omega(\delta),
$$

$$
0 \leqq j \leqq k-1
$$

where $z_{\nu}=\chi\left(e^{i \theta_{\nu}}\right)$.

It is readily shown by applying Theorems 2.4 and 2.5 to $d^{k} s(\theta) / d \theta^{k}$, when the latter is expressed in terms of the derivatives of $\zeta(\theta)$ and $\eta(\theta)$, that $d^{k} s / d \theta^{k}$ has modulus of continuity on $0 \leqq \theta \leqq 2 \pi$ less than $M_{4} \omega(\delta)$. This fact together with Theorem 2.5 and inequalities (2.3) yields the desired result when $h^{(k)}(\theta)$ is expressed in terms of $d^{i} s / d \theta^{j}$ and $\partial^{i} F / \partial s^{j}, j=1, \cdots, k$.

To relate the moduli of continuity of $F^{(k)}(z)$ and $\partial^{k} F / \partial s^{k}$ we have

THEOREM 2.7. Let $C$ be a rectifiable Jordan curve such that for every arc of $C$ the ratio of the length of that arc to the length of its corresponding chord is less than a constant $A$. Let $z=t(s)$ be the equation of $C$; suppose $t^{(k)}(s)$, $k \geqq 1$, is continuous with modulus of continuity not greater than $\omega(\delta)$. Let $F(z)$ be defined on $C$. Then a necessary and sufficient condition that $F^{(k)}(z)$ exist and be continuous on $C$ with modulus of continuity not greater than $L \omega(\delta)$ is that $\partial^{k} F / \partial s^{k}$ exist and be continuous on $C$ with modulus of continuity not greater than $L_{1} \omega(\delta)$.

Theorem 2.7 is readily established using methods employed above.

3. Problem $\alpha$, direct. Tchebycheff approximation. By a harmonic polynomial $p_{n}(z)$ of degree $n$ we shall mean the real part of a polynomial in $z$ of degree $n$. The degree of a polynomial will always be indicated by its subscript. None of the coefficients of $p_{n}(z)$ is assumed to be different from zero. When polar coordinates $(r, \theta)$ are introduced in the $z$-plane, the problem of approximation on the unit circle by harmonic polynomials becomes a problem in trigonometric approximation. A trigonometric sum of order $n, T_{n}(\theta)$ $=\sum_{k=0}^{n}\left(a_{k} \cos k \theta+b_{k} \sin k \theta\right)$, may be regarded as the boundary values on $|z|=1$ of a harmonic polynomial $p_{n}(z)$ of degree $n$, and conversely.

THeOREM 3.1. Let $C$ consist of a finite number of mutually exterior analytic Jordan curves. Let $u(z)$ be harmonic in the interior of $C$ and continuous on $\bar{C}$. Suppose $\partial^{k} u(z) / \partial s^{k}$ exists and is continuous with modulus of continuity $\omega(\delta)$ on $C, \omega(\delta) \not \equiv 0$. Then there exist harmonic polynomials $p_{n}(z)$ such that

$$
\left|u(z)-p_{n}(z)\right| \leqq M \frac{\omega(1 / n)}{n^{k}},
$$


When we refer to a function of $z$ which is harmonic or analytic in several mutually exterior regions, we do not imply that there is any relationship between the values of the function for $z$ in different regions. Here and throughout we write "there exist polynomials" rather than "for each $n, n=1,2, \cdots$, there exists a polynomial of degree $n$."

The case that $\partial^{k} u / \partial s^{k}$ satisfies a Lipschitz condition of order $\alpha$ on $C$ is treated in [11]. The method used below to prove Theorem 3.1 is a modification of one due to Curtiss [2]. Curtiss mentions without elaborating that his method may be used for analytic functions in connection with the modulus of continuity.

We now prove Theorem 3.1. Let $C$ consist of the Jordan curves $C^{\nu}$, $\nu=1, \cdots, \lambda$. Let $z=\chi_{\nu}(w), w=r e^{i \theta}$, map the interior of $|w|=1$ one-to-one and conformally onto the interior of $C^{\nu}$; set $u\left[\chi_{\nu}\left(e^{i \theta}\right)\right]=U_{\nu}(\theta)$. It follows by Theorem 2.6 that $U_{\nu}^{(\mathbf{k})}(\theta)$ has modulus of continuity on $0 \leqq \theta \leqq 2 \pi$ less than $M_{1} \omega(\delta), \nu=1, \cdots, \lambda$. It is known (see Achyèser's [1] formulation of certain of Jackson's results) that there exist constants $d_{m, j}$ which depend only on $j, m$, and $k$ such that

$$
\left|U_{\nu}(\theta)-\sum_{j=0}^{m} d_{m, j}\left(a_{j}^{\prime} \cos j \theta+b_{j}^{\nu} \sin j \theta\right)\right| \leqq \frac{L \omega(1 / m)}{m^{k}},
$$

where $a_{j}^{\nu}, b_{j}^{\nu}$ are the Fourier coefficients of $U_{\nu}(\theta)$. Define $P_{\nu, m}(w), m=1,2, \cdots$, as the harmonic polynomial of degree $m$ which is identical on $|w|=1$ with $\sum_{j=0}^{m} d_{m, j}\left(a_{j}^{\nu} \cos j \theta+b_{j}^{\nu} \sin j \theta\right)$. If $P_{\nu, m}(w)=F_{\nu, m}(z)$, we then have

$$
\left|u(z)-F_{v, m}(z)\right| \leqq M_{2} \frac{\omega(1 / m)}{m^{k}},
$$

$z$ on $\bar{C}^{p}$.

Let $w=\Omega_{\nu}(z)$ denote the inverse of $z=\chi_{\nu}(w)$. Since the curves $C^{v}$ are analytic and mutually exterior, there exists an $R>1$ such that the curves $\Gamma^{p}:\left|\Omega_{\nu}(z)\right|$ $=R$ are analytic and mutually exterior and such that $\Omega_{\nu}(z)$ is analytic and schlicht in $\bar{\Gamma}^{\nu}, \nu=1, \cdots, \lambda$.

Let $Q_{\nu, m}(w)=G_{\nu, m}(z)$ denote the conjugate of $P_{\nu, m}(w)=F_{\nu, m}(z), Q_{\nu, m}(0)$ $=0$. It follows from (3.1) that the $\left|P_{v, m}(w)\right|$ are uniformly bounded on $|w|=1$; hence by a known result [11, Theorem 9.6] we have

$$
\left|F_{r, m}(z)+i G_{v, m}(z)\right| \leqq M_{3} R^{m},
$$

$z$ on $\Gamma^{\nu}$.

Define $H_{m}(z) \equiv F_{\nu, m}(z)+i G_{\boldsymbol{v}, m}(z)$ for $z$ in $\bar{\Gamma}^{\nu}, \nu=1, \cdots, \lambda$. By the method used in [11, Theorem 8.1] we obtain that there exist polynomials $\pi_{q m}^{*}(z)$ in $z$, $m=1,2, \cdots$, such that

$$
\left|H_{m}(z)-\pi_{q m}^{*}(z)\right| \leqq M_{4} r^{m}, \quad z \text { in } \bar{C},
$$

where $q$ is a fixed positive integer and $r$ is a constant, $0<r<1$. Set $p_{q m}^{*}(z)$ $=\Re\left[\pi_{q m}^{*}(z)\right]$. (We denote by $\Re[F(z)]$ the real part of $F(z)$ and by $\Im[F(z)]$ the 
imaginary part of $F(z)$ divided by $i$.) Then inequalities (3.1) and (3.2) yield

$$
\left|u(z)-p_{q m}^{*}(z)\right| \leqq M_{2} \frac{\omega(1 / m)}{m^{k}}+M_{4} r^{m} \leqq M_{5} \frac{\omega(1 / m)}{m^{k}}, \quad z \text { on } \bar{C},
$$

since by virtue of Theorem $2.4, r^{m}<M_{6} / m^{k+1}<M_{7} \omega(1 / m) / m^{k}$.

Define the polynomials $p_{n}(z)$ in the statement of the theorem as $p_{n}(z) \equiv 0$ for $n=1,2, \cdots, q-1$, and $p_{q j+h}(z) \equiv p_{q j}^{*}(z)$ for $h=0,1, \cdots, q-1 ; j=1$, $2, \cdots$. There exists a constant $M_{8}$ independent of $j$ such that

$$
\frac{\omega(1 / j)}{j^{k}} \leqq M_{8} \frac{\omega(1 /(q j+h))}{(q j+h)^{k}},
$$

for using Theorem 2.3 we have

$$
\frac{\omega(1 / j)}{j^{k}} \cdot \frac{(q j+h)^{k}}{\omega(1 /(q j+h))}<\left(L_{1} \frac{q j+h}{j}+1\right)\left(\frac{q j+h}{j}\right)^{k}<M_{8}
$$

Hence Theorem 3.1 is established.

Lemma 3.2. Let $f(z)$ be analytic in $|z|<1$ and continuous in $|z| \leqq 1$. Suppose $f^{(k)}(z)$ exists and is continuous with modulus of continuity $\omega(\delta)$ on $|z|=1$, $\omega(\delta) \not \equiv 0$. Then there exist polynomials $\pi_{n}(z)$ in $z$ such that

$$
\left|f(z)-\pi_{n}(z)\right| \leqq M \frac{\omega(1 / n)}{n^{k}}, \quad|z| \leqq 1 .
$$

Here and in following similar situations it is sufficient to assume the existence of $f^{(k)}(z)$ on the boundary merely in the one-dimensional sense.

Set $f(z)=u(z)+i v(z)$. It follows by Theorem 2.7 that $\partial^{k} u\left(e^{i \theta}\right) / \partial \theta^{k}$ and $\partial^{k} v\left(e^{i \theta}\right) / \partial \theta^{k}, z=r e^{i \theta}$, have moduli of continuity on $0 \leqq \theta \leqq 2 \pi$ less than $M_{1} \omega(\delta)$. If the Fourier coefficients of $u\left(e^{i \theta}\right)$ are $a_{j}, b_{j}$, then those of $v\left(e^{i \theta}\right)$ are $-b_{j}, a_{j}$, where we assume without loss of generality that $v(0)=b_{0}=0$; hence in the notation of Theorem 3.1 we have

$$
\left|u\left(e^{i \theta}\right)+i v\left(e^{i \theta}\right)-\sum_{j=0}^{n} d_{n, j}\left(a_{i}-i b_{j}\right) e^{i j \theta}\right| \leqq L \frac{\omega(1 / n)}{n^{k}} .
$$

Lemma 3.2 follows if we define $\pi_{n}(z)$ as $\sum_{j=0}^{n} d_{n, j}\left(a_{j}-i b_{j}\right) z^{j}$.

Lemma 3.2 and the method used to establish Theorem 3.1 yield

THEOREM 3.3. Let $C$ consist of a finite number of mutually exterior analytic Jordan curves. Let $f(z)$ be analytic in the interior of $C$ and continuous in $\bar{C}$. Suppose $f^{(k)}(z)$ exists and is continuous on $C$ with modulus of continuity $\omega(\delta)$, $\omega(\delta) \not \equiv 0$. Then there exist polynomials $\pi_{n}(z)$ in $z$ such that

$$
\left|f(z)-\pi_{n}(z)\right| \leqq M \frac{\omega(1 / n)}{n^{k}}, \quad \quad z \text { on } \bar{C} .
$$


4. Problem $\alpha$, direct. Integral measures of approximation. Let $C$ be a rectifiable Jordan curve in the complex $z$-plane. Let $w=\Omega(z)$, with inverse $z=\chi(w)$, map the interior of $C$ one-to-one and conformally onto the interior of the circle $|w|=1$, where $w=r e^{i \theta}$; denote by $\Gamma_{r}$ the locus $|\Omega(z)|=r, r \leqq 1$. Let $u(z)$ be harmonic inside $C$. Suppose $p>1$ and

$$
\int_{\Gamma_{r}}|u(z)|^{p} d s \leqq M, \quad 0<r<1,
$$

where $M$ is independent of $r$. Set $u[\chi(w)]=U(w)$. If $\left|\Omega^{\prime}(z)\right| \leqq L$ for $z$ inside $C$, it follows that

$$
\int_{0}^{2 \pi}\left|U\left(r e^{i \theta}\right)\right|^{p} d \theta=r^{-1} \int_{\Gamma_{r}}|u(z)|^{p}\left|\Omega^{\prime}(z)\right| d s \leqq L_{1} M, \quad 0<r<1 .
$$

By a well known result we thus have that for almost all $\theta, U(w)$ approaches a limit $U\left(e^{i \theta}\right)$ as $w$ approaches $e^{i \theta}$ in angle from the interior of $|w|=1$, and $U\left(e^{i \theta}\right) \in L^{p}$ on $0 \leqq \theta \leqq 2 \pi$. Since at almost every point of $|w|=1$ the transformation $z=\chi(w)$ is conformal [5, pp. 85-87], it follows that the limit $u(\zeta)$ of $u(z)$ as $z$ approaches $\zeta$ in angle from the interior of $C$ exists for almost every point $\zeta$ of $C$. Furthermore, $|u(\zeta)|^{p}$ is summable Lebesgue on $C$.

We shall use frequently the following well known inequality:

$$
|\zeta+\eta|^{p} \leqq M\left(|\zeta|^{p}+|\eta|^{p}\right),
$$

where $M$ depends only on $p$.

To aid in the establishment of further direct results for Problem $\alpha$ we prove

TheOREM 4.1. Let $C$ be a rectifiable Jordan curve and let $z_{0}$ be a point inside $C$; let $z=\chi(w)$ map $|w|<1$ onto the interior of $C$ so that $z_{0}=\chi(0)$. Suppose that for all $w_{1}$ and $w_{2}$ on $|w|=1$ we have

$$
L_{1}\left|w_{1}-w_{2}\right| \leqq\left|\chi\left(w_{1}\right)-\chi\left(w_{2}\right)\right| \leqq L_{2}\left|w_{1}-w_{2}\right| .
$$

Let $u(z)$ be harmonic in the interior of $C$ and suppose $\int_{\Gamma_{r}}|u(z)|^{p} d s \leqq M, 0<r<1$, where $p>1$. If $f(z)=u(z)+i v(z)$, where $v(z)$ is conjugate to $u(z), v\left(z_{0}\right)=0$, then

$$
\begin{gathered}
\int_{C}|f(z)|^{p} d s \leqq\left(L_{2} / L_{1}\right) M_{p} \int_{C}|u(z)|^{p} d s, \\
\iint_{\bar{C}}|f(z)|^{p} d A \leqq\left(L_{2} / L_{1}\right)^{2} M_{p} \iint_{\bar{C}}|u(z)|^{p} d A,
\end{gathered}
$$

where $M_{p}$ depends only on $p$.

In Theorem 4.1 and following similar situations we suppose $f(z)$ to be defined for $z$ on $C$ as the limit of $f(\zeta)$ as $\zeta$ approaches $z$ in angle from the interior 
of $C$. Under the hypothesis of Theorem 4.1 this limit exists and is independent of the path of approach for almost all $z$ on $C$, as will appear from the proof of Theorem 4.1.

Inequality (4.3) has been established for the unit circle by M. Riesz [6, p. 225].

Under the hypothesis on $C$, it is known (see for example [7, Theorem 1.2.7]) that inequality (4.2) is valid for $\left|w_{1}\right| \leqq 1,\left|w_{2}\right| \leqq 1$. Hence it follows that $L_{1} \leqq\left|\chi^{\prime}(w)\right| \leqq L_{2}$ for $|w|<1$, and $\left|\Omega^{\prime}(z)\right| \leqq 1 / L_{1}$ for $z$ inside $C$. Thus $u\left[\chi\left(e^{i \theta}\right)\right]$ $\in L^{p}$ on $0 \leqq \theta \leqq 2 \pi$; by Riesz's result [6, p. 225] and (4.1) we therefore have that for almost all $\theta, f[\chi(w)]$ approaches a limit $f\left[\chi\left(e^{i \theta}\right)\right]$ as $w$ approaches $e^{i \theta}$ in angle and

$$
\int_{0}^{2 \pi}\left|f\left[\chi\left(e^{i \theta}\right)\right]\right| p d \theta \leqq M_{p} \int_{0}^{2 \pi}\left|u\left[\chi\left(e^{i \theta}\right)\right]\right| p d \theta
$$

where $M_{p}$ depends only on $p$.

From the hypothesis on $C$ it follows (see for example $[11, \S 9]$ ) that almost everywhere on $|w|=1, \chi^{\prime}(w)$ exists and $L_{1} \leqq\left|\chi^{\prime}(w)\right| \leqq L_{2}$. Suppose arclength $s$ measured along $C$ has equation $s=s(\theta)$. Then $[11, \S 9]$ almost everywhere on $|w|=1$ we have $s^{\prime}(\theta)=\left|\chi^{\prime}(w)\right|$; hence by virtue of (4.5) we have $[3$, p. 467]

$$
\begin{aligned}
\int_{C}|f(z)|^{p} d s & =\int_{0}^{2 \pi}\left|f\left[\chi\left(e^{i \theta}\right)\right]\right|{ }^{p} s^{\prime}(\theta) d \theta \leqq L_{2} \int_{0}^{2 \pi}\left|f\left[\chi\left(e^{i \theta}\right)\right]\right| p d \theta \\
& \leqq L_{2} M_{p} \int_{0}^{2 \pi}\left|u\left[\chi\left(e^{i \theta}\right)\right]\right|^{p} d \theta \leqq\left(L_{2} / L_{1}\right) M_{p} \int_{C}|u(z)|^{p} d s .
\end{aligned}
$$

Inequality (4.3) is established.

It follows by (4.3) that

$$
\begin{aligned}
\iint_{\bar{c}}|f(z)|^{p} d A & \leqq L_{2}^{2} \int_{0}^{1} r d r \int_{0}^{2 \pi}\left|f\left[\chi\left(r e^{i \theta}\right)\right]\right|{ }^{p} d \theta \\
& \leqq L_{2}^{2} M_{p} \int_{0}^{1} r d r \int_{0}^{2 \pi}\left|u\left[\chi\left(r e^{i \theta}\right)\right]\right|^{p} d \theta \\
& \leqq\left(L_{2} / L_{1}\right)^{2} M_{p} \iint_{\bar{C}}|u(z)|^{p} d A .
\end{aligned}
$$

The proof of Theorem 4.1 is complete.

THEOREM 4.2. Let $C$ consist of a finite number of mutually exterior analytic Jordan curves. Let $u(z)$ be harmonic in the interior of $C$ and continuous on $\bar{C}$; set $f(z)=u(z)+i v(z)$, where $v(z)$ is conjugate to $u(z)$. Suppose $\partial^{k} u(z) / \partial s^{k}$ is continuous with modulus of continuity $\omega(\delta)$ on $C, \omega(\delta) \not \equiv 0$. Then there exist 
polynomials $\pi_{n}(z)$ in $z$ such that

$$
\begin{gathered}
\int_{C} \rho(s)\left|f(z)-\pi_{n}(z)\right|^{p} d s \leqq M\left[\frac{\omega(1 / n)}{n^{k}}\right]^{p}, \\
\iint_{\bar{C}} \rho_{1}(z)\left|f(z)-\pi_{n}(z)\right|^{p} d A \leqq M_{0}\left[\frac{\omega(1 / n)}{n^{k}}\right]^{p},
\end{gathered}
$$

where $\rho(s)$ is a bounded, measurable, non-negative function of $s ; \rho_{1}(z)$ is bounded, measurable, and non-negative for $z$ on $\bar{C}$; and $p>0$.

We establish in detail only (4.6); the same method of proof establishes (4.7), provided we substitute in the proof use of (4.4) for use of (4.3).

By Theorem 3.1 there exist harmonic polynomials $p_{m}(z)$ such that

$$
\left|u(z)-p_{m}(z)\right| \leqq L \frac{\omega(1 / m)}{m^{k}},
$$

Let $C$ be composed of the mutually exterior curves $C^{\nu}, \nu=1, \cdots, \lambda$. Let $z_{\nu}$ be a point interior to $C^{\nu}$; set $P_{m, v}(z)=p_{m}(z)+i q_{m, v}(z)$, where $q_{m, v}(z)$ is conjugate to $p_{m}(z), q_{m, \nu}\left(z_{v}\right)=v\left(z_{v}\right)$. If $p>1$, by Theorem 4.1 and (4.8) we have

$$
\begin{aligned}
\int_{c^{\nu}}\left|f(z)-P_{m, \nu}(z)\right|^{p} d s & \leqq M_{1} \int_{c^{\nu}}\left|u(z)-p_{m}(z)\right|^{p} d s \\
& \leqq M_{2}\left[\frac{\omega(1 / m)}{m^{k}}\right]^{p} .
\end{aligned}
$$

If $0<p \leqq 1$, from Hölder's inequality and inequality (4.9) we obtain

$$
\begin{aligned}
\int_{c^{\nu}}\left|f(z)-P_{m, \nu}(z)\right|^{p} d s & \leqq M_{3}\left[\int_{c^{\nu}}\left|f(z)-P_{m, \nu}(z)\right|^{2} d s\right]^{p / 2} \\
& \leqq M_{4}\left[\omega(1 / m) / m^{k}\right]^{p} .
\end{aligned}
$$

Choose $R>1$ such that the curves $C_{R}^{\nu}, \nu=1, \cdots, \lambda$, are mutually exterior. ( $C_{R}^{\nu}$ denotes, in conformity with our usual notation, the locus $\left|\phi_{\nu}(z)\right|$ $=R$, where $w=\phi_{\nu}(z)$ maps the exterior of $C^{\nu}$ onto $|w|>1$ so that the points at infinity in the two planes correspond.) It follows from (4.8) that $\left|p_{m}(z)\right|$ $\leqq M_{5}$ for $z$ on $\vec{C}$; hence by a known result $\left[11\right.$, Theorem 9.6] we have $\mid P_{m, v}(z)$ $-v\left(z_{\nu}\right) \mid \leqq M_{6} R^{m}$ and thus $\left|P_{m, \nu}(z)\right| \leqq M_{7} R^{m}$ for $z$ on $C_{R}^{\nu}$. Set $F_{m}(z) \equiv P_{m, v}(z)$, $z$ in $\bar{C}_{R}^{\nu}, \nu=1, \cdots, \lambda$. By the method used in [11, Theorem 8.1] we obtain that there exist polynomials $\pi_{q m}^{*}(z)$ in $z, m=1,2, \cdots$, such that

$$
\left|F_{m}(z)-\pi_{q m}^{*}(z)\right| \leqq M_{8} r_{0}^{m}, \quad z \text { in } \bar{C},
$$
where $q$ is a fixed positive integer and $r_{0}$ is a constant, $0<r_{0}<1$. Inequalities (4.1) and (4.9)-(4.11) yield 


$$
\int_{C} \rho(s)\left|f(z)-\pi_{q m}^{*}(z)\right| p d s \leqq M_{9}\left[\frac{\omega(1 / m)}{m^{k}}\right]^{p}+M_{10} r_{0}^{m p} .
$$

The conclusion of Theorem 4.2 now follows (cf. the proof of Theorem 3.1) if we define the polynomials $\pi_{n}(z)$ in the statement of the theorem as $\pi_{n}(z) \equiv 0$, $n=1, \cdots, q-1$ and $\pi_{q j+h}(z) \equiv \pi_{q j}^{*}(z), h=0,1, \cdots, q-1 ; j=1,2, \cdots$.

5. Problem $\alpha$, indirect. Tchebycheff theorems. In the preceding two sections from given continuity properties of a harmonic or analytic function we have derived results on degree of convergence to the function of a sequence of approximating polynomials. In this section we study the converse question for approximation in the sense of Tchebycheff. We establish our theorems for a single closed Jordan region, but the same theorems clearly hold for a finite number of such regions which are mutually exterior.

Definition 5.1. A curve $C$ is said to be of Type $\mathrm{D}$ if (i) $C$ is a rectifiable Jordan curve such that for every arc of $C$ the ratio of the length of that arc to the length of its corresponding chord is less than a constant $A$; and (ii) there exists a number $\delta_{0}>0$ such that through any point $P$ of $C$ there is a circle of radius $\delta_{0}$ whose closed interior lies in $\bar{C}$.

We shall need the following two theorems [11, Theorems $7.2,7.4]$, the first of which is essentially due to Szegö:

TheORem 5.1. Let $C$ be a curve of Type D; let $\pi_{n}(z)$ be a polynomial in $z$ of degree $n$. If $\left|\Re\left[\pi_{n}(z)\right]\right| \leqq K$, z on $C$, then $\left|\pi_{n}^{\prime}(z)\right| \leqq K n / \delta_{0}, z$ in $\bar{C}$, where $\delta_{0}$ is the constant of Definition 5.1 .

THEOREM 5.2. Under the hypothesis of Theorem 5.1 we have

$$
\left|\pi_{n}\left(z_{1}\right)-\pi_{n}\left(z_{2}\right)\right| \leqq A K n\left|z_{1}-z_{2}\right| / \delta_{0}, \quad z_{1}, z_{2} \text { on } \bar{C},
$$

where $A$ and $\delta_{0}$ are the constants of Definition 5.1 .

We henceforth assume $\Omega(x)$ to be a real, non-negative function which is nonincreasing for $x$ sufficiently large and which is such that $\int^{\infty}[\Omega(x) / x] d x$ exists.

The method to be used in proving the following theorem is a modification of one employed by de la Vallée Poussin [4, chap. IV] to establish results on trigonometric approximation.

Theorem 5.3. Let $u(z)$ be defined in $\bar{C}$, where $C$ is a curve of Type D; suppose harmonic polynomials $p_{n}(z)$ exist such that

$$
\left|u(z)-p_{n}(z)\right| \leqq \Omega(n) / n^{k},
$$

z in $\bar{C}$.

Then $u(z)$ is harmonic in the interior of $C$ and continuous on $\bar{C}$ with modulus of continuity $\omega(\delta)$ which satisfies the condition

$$
\omega(\delta) \leqq L\left[\delta \int_{a}^{a / \delta} \Omega(x) d x+\int_{1 / \delta}^{\infty} \frac{\Omega(x)}{x} d x\right], \quad 0<\delta \leqq 1 / a,
$$


where $a>1$ is a constant independent of $\delta$. Set $f(z)=u(z)+i v(z)$, where $v(z)$ is conjugate to $u(z)$. If $k \geqq 1$, then $f^{(k)}(z)$ exists and is continuous on $\bar{C}$ with modulus of continuity which satisfies a condition of form (5.2).

The harmonicity interior to $C$ and continuity on $\bar{C}$ of $u(z)$ follow from (5.1). Let $a>1$ be an integer such that $\Omega(x)$ is nonincreasing for $x>a$. By use of the triangle inequality and (5.1) we obtain, for $j=1,2, \cdots$,

$$
\left|p_{a^{j+1}}(z)-p_{a^{j}}(z)\right| \leqq 2 \Omega\left(a^{j}\right) / a^{j k}, \quad z \text { in } \bar{C} .
$$

We write

$$
u(z)=p_{a^{2}}(z)+\sum_{j=2}^{\mu}\left[p_{a^{j+1}}(z)-p_{a^{j}}(z)\right]+\sum_{j=\mu+1}^{\infty}\left[p_{a^{i+1}}(z)-p_{a^{i}}(z)\right], \quad z \text { in } \bar{C},
$$

where $\mu$ is a positive integer to be determined later.

Let $\zeta$ be a fixed point on $C$ such that $f(\zeta)$ is defined; set $\pi_{n}(z)=p_{n}(z)+i q_{n}(z)$, $q_{n}(\zeta)=v(\zeta)$. Since $\left|p_{a^{2}}(z)\right| \leqq M$ for $z$ in $\bar{C}$, it follows from Theorems 5.1 and 5.2 that for $\nu=0, k$,

$$
\left|\pi_{a^{2}}^{(\nu)}\left(z_{1}\right)-\pi_{a^{2}}^{(\nu)}\left(z_{2}\right)\right| \leqq M_{1}\left|z_{1}-z_{2}\right|, \quad z_{1} \text { and } z_{2} \text { on } \bar{C} .
$$

By the use of inequality (5.3) and Theorems 5.1 and 5.2 we obtain for $z_{1}$ and $z_{2}$ on $\bar{C}$ and $\nu=0, k$ :

$$
\begin{aligned}
& \left|\left[\pi_{a^{j+1}}^{(\nu)}\left(z_{1}\right)-\pi_{a^{j}}^{(\nu)}\left(z_{1}\right)\right]-\left[\pi_{a^{j+1}}^{(\nu)}\left(z_{2}\right)-\pi_{a^{j}}^{(\nu)}\left(z_{2}\right)\right]\right| \leqq M_{2} \Omega\left(a^{j}\right) a^{j}\left|z_{1}-z_{2}\right| ; \\
& \sum_{j=2}^{\mu}\left|\left[\pi_{a^{j+1}}^{(\nu)}\left(z_{1}\right)-\pi_{a^{j}}^{(\nu)}\left(z_{1}\right)\right]-\left[\pi_{a^{j+1}}^{(\nu)}\left(z_{2}\right)-\pi_{a^{j}}^{(\nu)}\left(z_{2}\right)\right]\right| \\
& \quad \leqq M_{3}\left|z_{1}-z_{2}\right| \sum_{j=2}^{\mu} \Omega\left(a^{j}\right)\left(a^{j}-a^{j-1}\right) \leqq M_{3}\left|z_{1}-z_{2}\right| \int_{a}^{a^{\mu}} \Omega(x) d x .
\end{aligned}
$$

Inequality (5.3) yields for $z$ in $\bar{C}$ :

$$
\begin{aligned}
\sum_{j=\mu+1}^{\infty}\left|p_{a^{j+1}}(z)-p_{a^{i}}(z)\right| & \leqq \frac{2 a}{a-1} \sum_{j=\mu+1}^{\infty} \frac{\Omega\left(a^{j}\right)}{a^{j}}\left(a^{j}-a^{j-1}\right) \\
& \leqq M_{4} \int_{a^{\mu}}^{\infty} \frac{\Omega(x)}{x} d x .
\end{aligned}
$$

From (5.4), (5.6), and (5.7) we thus have, if $\omega(\delta)$ denotes the modulus of continuity of $u(z)$ in $\bar{C}$,

$$
\omega(\delta) \leqq M_{1} \delta+M_{3} \delta \int_{a}^{a^{\mu}} \Omega(x) d x+2 M_{4} \int_{a^{\mu}}^{\infty}[\Omega(x) / x] d x .
$$

Let $\delta$ be arbitrary, but fixed. Choose the integer $\mu$ such that $a^{\mu-1} \leqq 1 / \delta<a^{\mu}$; this is possible for all $\delta, 0<\delta \leqq 1 / a$. It then follows that $\omega(\delta)$ satisfies a rela- 
tion of form (5.2).

Now suppose $k \geqq 1$. Theorem 5.2 and inequality (5.3) yield for $z$ in $\bar{C}$ :

$$
\begin{gathered}
\left|\pi_{a^{j+1}}(z)-\pi_{a^{j}}(z)\right| \leqq\left|p_{a^{j+1}}(\zeta)-p_{a^{j}}(\zeta)\right|+M_{5} \Omega\left(a^{j}\right) a^{j+1}|z-\zeta| / a^{j k} \leqq M_{6} \Omega\left(a^{j}\right) ; \\
\sum_{j=2}^{\infty}\left|\pi_{a^{i+1}}(z)-\pi_{a^{i}}(z)\right| \leqq M_{7} \int_{a}^{\infty}[\Omega(x) / x] d x .
\end{gathered}
$$

Hence by virtue of (5.1) we have

$$
f(z)=\pi_{a^{2}}(z)+\sum_{j=2}^{\infty}\left[\pi_{a^{j+1}}(z)-\pi_{a^{j}}(z)\right], \quad z \text { in } \bar{C} .
$$

By the use of Theorem 5.1 and inequality (5.3) we likewise obtain for $\nu=1, \cdots, k$,

$$
\begin{array}{ll}
\left|\pi_{a^{i+1}}^{(\nu)}(z)-\pi_{a^{i}}^{(\nu)}(z)\right| \leqq M_{8} \Omega\left(a^{j}\right) a^{(j+1) \nu} / a^{j k} \leqq M_{9} \Omega\left(a^{j}\right), & z \text { in } \bar{C} ; \\
\sum_{j=\mu+1}^{\infty}\left|\pi_{a^{i+1}}^{(\nu)}(z)-\pi_{a^{j}}^{(\nu)}(z)\right| \leqq M_{10} \int_{a^{\mu}}^{\infty}[\Omega(x) / x] d x, & z \text { in } \bar{C},
\end{array}
$$

where $\mu$ is any positive integer. Thus

$$
\sum_{j=2}^{\infty}\left[\pi_{a^{j+1}}^{(\nu)}(z)-\pi_{a^{j}}^{(\nu)}(z)\right], \quad \nu=1, \cdots, k,
$$

converges uniformly in $\bar{C}$, and we have (cf. the treatment of a similar situation in $[11, \S 8])$

$$
f^{(k)}(z)=\pi_{a^{2}}^{(k)}(z)+\sum_{j=2}^{\mu}\left[\pi_{a^{i+1}}^{(k)}(z)-\pi_{a^{i}}^{(k)}(z)\right]+\sum_{j=\mu+1}^{\infty}\left[\pi_{a^{j+1}}^{(k)}(z)-\pi_{a^{j}}^{(k)}(z)\right] .
$$

The continuity of $f^{(k)}(z)$ in $\bar{C}$ is now evident. Let $\omega_{1}(\delta)$ denote the modulus of continuity of $f^{(k)}(z)$ on $\bar{C}$; it follows from inequalities (5.4), (5.6), and (5.9) that $\omega_{1}(\delta)$ satisfies a relation of form (5.8). We choose $\mu$ as before and the proof of Theorem 5.3 is complete.

Theorems 5.3 and 2.7 yield

THEOREM 5.4. Let the equation of $C b e z=t(s)$. In addition to the hypothesis of Theorem 5.3 suppose that $t^{(k)}(s)$ exists and is continuous with modulus of continuity which satisfies a condition of form (5.2). Then $\partial^{k} u(z) / \partial s^{k}$ exists and is continuous on $C$ with modulus of continuity which satisfies a condition of the same form.

To obtain Theorem 5.4 for $k \geqq 1$ the stronger hypothesis on $C$ is necessary, for suppose $C$ does not satisfy the additional hypothesis of Theorem 5.4. Then either $\Re\left[t^{(k)}(s)\right]$ or $\Im\left[t^{(k)}(s)\right]$ fails to be continuous with modulus of continuity which satisfies a condition of form (5.2). In the former case we take 
as a counterexample $u(z) \equiv p_{n}(z) \equiv x$ (where $\left.z=x+i y\right)$; in the latter case we take $u(z) \equiv p_{n}(z) \equiv y$.

THEOREM 5.5. In addition to the hypothesis of Theorem 5.3 [Theorem 5.4] suppose there exists an $\alpha<1$ such that $x^{\alpha} \Omega(x)$ is nondecreasing for $x>a_{0}$, where $\alpha$ and $a_{0}$ are constants. Then the conclusion of Theorem 5.3 [Theorem 5.4] is valid when inequality (5.2) is replaced by the inequality

$$
\omega(\delta) \leqq L_{1} \int_{1 / \delta}^{\infty} \frac{\Omega(x)}{x} d x, \quad 0<\delta \leqq \delta_{1},
$$

where $\delta_{1}$ is a suitable constant.

De la Vallée Poussin [4, pp. 56-57] has established the corresponding theorem for the trigonometric case by proving that the quotient

$$
x \int_{x}^{\infty} \frac{\Omega(x)}{x} d x / \int_{a_{0}}^{a_{0} x} \Omega(x) d x
$$

is bounded from zero as $x$ becomes infinite. Hence his proof is a valid one for Theorem 5.5. For certain theorems which follow we clearly have an extension of the nature of Theorem 5.5 ; the statement of such extensions will be left to the reader. From the results of $[11, \S 2]$ and the theorems just proved, one may easily establish continuity properties of the partial derivatives of $u(z)$ similar to those established in Theorem 5.3 for $f^{(k)}(z)$. D.

Indirect theorems may also be proved for curves not necessarily of Type

Definition 5.2. A rectifiable Jordan arc or curve $C$ is said to be of $T y p e \mathrm{t}$, $1 \leqq t \leqq 2$, if (i) for every arc of $C$ the ratio of the length of that arc to the length of its corresponding chord is less than a constant $A$; and (ii) for $R>1$ we have $\min \left|z-z^{*}\right| \geqq K(C)(R-1)^{t}$, where $z \in C, z^{*} \in C_{R}$, and $K(C)$ is a positive constant depending on $C$ but independent of $R$.

The method used to establish Theorem 5.3 together with known results [7, Theorem 2.1.4], [11, Theorems 2.1, 2.2] yield

ThEOREM 5.6. Let $f(z)$ be defined in $\bar{C}$, where $C$ is a rectifiable Jordan arc or curve of Type $\mathrm{t}$; suppose there exist polynomials $\pi_{n}(z)$ such that

$$
\left|f(z)-\pi_{n}(z)\right| \leqq \frac{\Omega\left(n^{t}\right)}{n^{k t}}, \quad \quad z \text { on } \bar{C} .
$$

Then $f(z)$ is analytic in the interior of $C$ and continuous on $\bar{C} ; f^{(k)}(z)$ exists and is continuous on $\bar{C}$ with modulus of continuity which satisfies a condition of form (5.2).

6. Problem $\alpha$, indirect. Integral measures of approximation. In indirect theorems for harmonic functions where degree of approximation is measured 
by a line integral, continuity properties of $u(z)$ are concluded from the existence of a sequence of harmonic polynomials $p_{n}(z)$ which satisfy a relation of the form

$$
\int_{C} \rho(s)\left|u(z)-p_{n}(z)\right|^{p} d s \leqq \epsilon_{n}^{p} .
$$

Since the Lebesgue integral is used, the value of $u(z)$ on a point set on $C$ of measure zero has no effect on $\epsilon_{n}$. Therefore unless $u(z)$ is assumed to be continuous on $C$, our results must concern the behavior of $u(z)$ almost everywhere rather than everywhere on $C$. A corresponding remark applies to the analytic case. As in $\S 5$, it is sufficient to consider only a single curve.

The following theorem on polynomials will be useful:

TheOREM 6.1. Let $C$ be a curve of Type $\mathrm{D}$; let $p_{n}(z)$ be a harmonic polynomial. If $p>0$, then

$$
\left|p_{n}(z)\right| \leqq L n^{1 / p}\left[\int_{C}\left|p_{n}(z)\right|^{p} d s\right]^{1 / p}, \quad \quad z \text { on } \bar{C},
$$

where $L$ depends only on $C$.

Set $\mu_{n}=\left[\max \left|p_{n}(z)\right|, z\right.$ on $\left.C\right]$; let $\zeta$ be a point of $C$ such that $\left|p_{n}(\zeta)\right|=\mu_{n}$. Then by Theorem 5.2 we have

$$
\left|p_{n}(z)-p_{n}(\zeta)\right| \leqq A n \mu_{n}|z-\zeta| / \delta_{0}, \quad z \text { on } \bar{C},
$$

where $A$ and $\delta_{0}$ are the constants of Definition 5.1. Hence $\left|p_{n}(z)\right|$ $\geqq \mu_{n}\left(1-A n|z-\zeta| / \delta_{0}\right), z$ on $\bar{C}$. Let $\sigma$ denote the closed arc of $C$ which contains $\zeta$ and whose end points are the two first points of intersection of $C$ and the circle $|z-\zeta|=\delta_{0} / 2 n A$ as one proceeds along $C$ from $\zeta$ in the positive and in the negative direction. Such points of intersection clearly exist. We then have that $\left|p_{n}(z)\right| \geqq \mu_{n} / 2$ for $z$ on $\sigma$. Hence

$$
\int_{C}\left|p_{n}(z)\right|^{p} d s \geqq \int_{\sigma}\left|p_{n}(z)\right|^{p} d s \geqq\left(\mu_{n} / 2\right)^{p} \delta_{0} / A n .
$$

Theorem 6.1 now follows.

Theorem 6.2. Let $U(z)$ be defined on $C$, where $C$ is a curve of Type D. Suppose there exist harmonic polynomials $p_{n}(z)$ such that

$$
\int_{C} \rho(s)\left|U(z)-p_{n}(z)\right|^{p} d s \leqq \frac{[\Omega(n)]^{p}}{n^{k p+1}}, \quad \quad p>0,
$$

where $\rho(s)$ is positive, integrable, and bounded from zero. Then $\lim _{n \rightarrow \infty} p_{n}(z)$, $z$ on $\bar{C}$, exists. If $u(z)=\lim _{n \rightarrow \infty} p_{n}(z), z$ on $\bar{C}$, then $U(z)=u(z)$ almost everywhere on $C ; u(z)$ is harmonic in the interior of $C$ and continuous on $\bar{C}$ with modulus 
of continuity $\omega(\delta)$ which satisfies a condition of form (5.2). Set $f(z)=u(z)+i v(z)$, where $v(z)$ is conjugate to $u(z)$. If $k \geqq 1$, then $f^{(k)}(z)$ exists and is continuous on $\bar{C}$ with modulus of continuity which satisfies a condition of form (5.2).

Let $a>1$ be an integer such that $\Omega(x)$ is nonincreasing for $x>a$. Since $\rho(s)$ is bounded from zero, it follows from (4.1) and (6.1) that

$$
\int_{C}\left|p_{a^{j+1}}(z)-p_{a^{j}}(z)\right|^{p} d s \leqq M\left[\Omega\left(a^{j}\right)\right]^{p} / a^{j(k p+1)} .
$$

By Theorem 6.1 we thus have

$$
\left|p_{a^{i+1}}(z)-p_{a^{j}}(z)\right| \leqq M_{1} \Omega\left(a^{j}\right) / a^{j k},
$$

$z$ in $\bar{C}$.

The method employed to prove Theorem 5.3 now yields the desired continuity properties of $u(z)$ and $f(z)$. That $u(z)$ when defined as $u=p_{a^{2}}$ $+\sum_{j=2}^{\infty}\left(p_{a^{i+1}}-p_{a^{i}}\right)$ is the same function as $\lim _{n \rightarrow \infty} p_{n}(z)$ follows from the inequality

$$
\left|u-p_{n}\right| \leqq\left|u-p_{a^{m}}\right|+\left|p_{a^{m}}-p_{n}\right| \leqq\left|u-p_{a^{m}}\right|+M_{2} \Omega(n) / n^{k},
$$

where $m$ is chosen so that $a^{m-1} \leqq n<a^{m}$. Clearly the last member of (6.2) approaches zero as $n$ becomes infinite.

By the use of (4.1) we obtain

$$
\int_{C}|U-u|^{p} d s \leqq M_{3} \int_{C}\left|U-p_{n}\right|^{p} d s+M_{3} \int_{C}\left|u-p_{n}\right|^{p} d s .
$$

The right side of (6.3) can be made arbitrarily small by choosing $n$ sufficiently large, and the left side is a constant; therefore $\int_{C}|U-u|^{p} d s=0$, and $U(z)=u(z)$ almost everywhere on $C$.

Theorems 2.7 and 6.2 yield

THEOREM 6.3. Let the equation of $C$ be $z=t(s)$. In addition to the hypothesis of Theorem 6.2 suppose that $t^{(k)}(s)$ exists and is continuous with modulus of continuity which satisfies a condition of form (5.2). Then $\partial^{k} u / \partial s^{k}$ exists and is continuous on $C$ with modulus of continuity which satisfies a condition of the same form.

The method used to establish Theorem 6.1 may be used to prove Then

TheOREM 6.4. Let $C$ be a curve of Type $\mathrm{D}$; let $\pi_{n}(z)$ be a polynomial in $z$.

$$
\left|\pi_{n}(z)\right| \leqq M n^{1 / p}\left[\int_{C}\left|\pi_{n}(z)\right|^{p} d s\right]^{1 / p}, \quad \text { zon } \bar{C}, p>0 .
$$

Theorem 6.4 together with the method of proof of Theorem 6.2 yields a result that treats the case $k=0$ more fully than does Theorem 6.2: 
THEOREM 6.5. Let $C$ and $\rho(s)$ satisfy the hypothesis of Theorem 6.2. Let $F(z)$ be defined on $C$ and suppose there exist polynomials $\pi_{n}(z)$ in $z$ such that

$$
\int_{C} \rho(s)\left|F(z)-\pi_{n}(z)\right|^{p} d s \leqq[\Omega(n)]^{p} / n^{k p+1}, \quad \text { where } p>0 .
$$

Set $f(z)=\lim _{n \rightarrow \infty} \pi_{n}(z), z$ in $\bar{C}$. Then $f(z)=F(z)$ almost everywhere on $C ; f(z)$ is analytic in the interior of $C$ and continuous on $\bar{C} ; f^{(k)}(z)$ exists and is continuous in $\bar{C}$ with modulus of continuity $\omega(\delta)$ which satisfies a condition of form (5.2).

The methods used in proving Theorem 5.7 and Theorem 6.2 together with the analogue of Theorem 6.4 for curves of Type $t$ [7, Theorem 2.2.1] may be used to establish

TheOREM 6.6. Let $F(z)$ be defined on $C$, where $C$ is a curve of Type $\mathrm{t}$; if polynomials $\pi_{n}(z)$ in $z$ exist such that

$$
\int_{C} \rho(s)\left|F(z)-\pi_{n}(z)\right|^{p} d s \leqq \frac{\left[\Omega\left(n^{t}\right)\right]^{p}}{n^{(k p+1) t}},
$$

where $\rho(s)$ is positive, integrable, and bounded from zero and $p>0$, then the conclusion of Theorem 6.5 is valid.

For surface integrals we prove

TheOREM 6.7. Let $C$ be a curve of Type D. If $p_{n}(z)$ is a harmonic polynomial, then

$$
\left|p_{n}(z)\right| \leqq L n^{2 / p}\left[\iint_{\bar{C}}\left|p_{n}(z)\right|^{p} d A\right]^{1 / p}, \quad \text { zon } \bar{C}, p>0 .
$$

The proof of Theorem 6.7 is similar to that of Theorem 6.1. If as in Theorem 6.1 we set $\mu_{n}=\left[\max \left|p_{n}(z)\right|, z\right.$ on $\left.C\right]$ and let $\zeta$ be a point of $C$ such that $\left|p_{n}(\zeta)\right|=\mu_{n}$, then

$$
\left|p_{n}(z)\right| \geqq \mu_{n}\left(1-A n|z-\zeta| / \delta_{0}\right), \quad z \text { on } \bar{C} .
$$

Under the hypothesis on $C$ there is a circle $\Gamma$ through $\zeta$ of radius $\delta_{0} / 4 n A$ whose closed interior lies in $\bar{C}$. It follows that in $\bar{\Gamma}$ we have $\left|p_{n}(z)\right| \geqq \mu_{n} / 2$. Hence

$$
\iint_{\bar{C}}\left|p_{n}(z)\right|^{p} d A \geqq \iint_{\bar{\Gamma}}\left|p_{n}(z)\right|^{p} d A \geqq\left(\mu_{n} / 2\right)^{p} \pi\left(\delta_{0} / 4 n A\right)^{2} .
$$

Theorem 6.7 now follows.

To show that for $p=2$ the exponent of $n$ cannot be improved in Theorem 6.7 we take $C$ as the unit circle and $p_{n}(z)=\sum_{j=0}^{n} r^{j} \cos j \theta$.

Theorem 6.7 together with the method used to prove Theorem 6.2 yields 
TheOREM 6.8. Let $C$ be a curve of Type D. Let $U(z)$ be defined in $\bar{C}$; suppose there exist harmonic polynomials $p_{n}(z)$ such that

$$
\iint_{\bar{c}} \rho(z)\left|U(z)-p_{n}(z)\right|^{p} d A \leqq \frac{[\Omega(n)]^{p}}{n^{k p+2}}, \quad p>0,
$$

where $\rho(z)$ is positive, integrable, and bounded from zero on $\bar{C}$. Then $\lim _{n \rightarrow \infty} p_{n}(z)$, $z$ on $\bar{C}$, exists. If $u(z)=\lim _{n \rightarrow \infty} p_{n}(z), z$ on $\bar{C}$, then $U(z)=u(z)$ almost everywhere on $\bar{C}$, and the conclusion of Theorem 6.2 is valid.

The analogue of Theorem 6.7 for a polynomial $\pi_{n}(z)$ in $z$ can clearly be proved by the method used to prove Theorem 6.7. We also have for surface integrals theorems corresponding to Theorems 6.3 and 6.5. The statement and proof of these theorems are left to the reader.

7. Problem $\beta$, direct. Let $C$ consist of a finite number of mutually exterior Jordan curves. Let $z=\psi(w)$ map $|w|>1$ conformally onto the complement of $\bar{C}$ so that the points at infinity in the two planes correspond to each other. Walsh and Sewell [10] make the following definition:

Definition 7.1. The set $C$ is called a contour if $C$ consists of mutually exterior Jordan curves $C_{1}, C_{2}, \cdots, C_{\lambda}$ having the properties (i) each $C_{j}$ has a tangent at every point; (ii) the function

$$
\log \left|\frac{\psi(w)-\psi\left(w^{\prime}\right)}{w-w^{\prime}}\right|
$$

for every possible definition of $\psi(w)$ and $\psi\left(w^{\prime}\right)$ is locally bounded in the twodimensional sense for $|w|=1$ and $\left|w^{\prime}\right| \geqq 1$ in the neighborhood of each point of continuity of $\psi(w)$; (iii) the function (7.1) satisfies on each arc $\gamma_{j}$ of $|w|=1$ corresponding to a curve $C_{j}$ of $C$ a Lipschitz condition of order unity in w, uniformly with respect to $w^{\prime}$ on any closed arc of $\left|w^{\prime}\right|=1$ interior to $a \gamma_{k}$.

As a direct theorem for Problem $\beta$ we prove

Theorem 7.1. Let $C$ and $C_{R}, R>1$, be contours. Let $u(z)$ be harmonic in the interior of $C_{R}$ and continuous on $\bar{C}_{R} ;$ suppose $\partial^{k} u(z) / \partial s^{k}$ exists and is continuous with modulus of continuity $\omega(\delta)$ on $C_{R}, \omega(\delta) \neq \equiv 0$. Set $f(z)=u(z)+i v(z)$, where $v(z)$ is conjugate to $u(z)$. Then there exist polynomials $\pi_{n}(z)$ in $z$ such that

$$
\left|f(z)-\pi_{n}(z)\right| \leqq M \frac{\omega(1 / n)}{n^{k} R^{n}}, \quad z \text { in } \bar{C} \text {. }
$$

By Theorem 4.2 there exist polynomials $P_{n}(z)$ in $z$ such that

$$
\int_{C_{R}}\left|f(z)-P_{n}(z)\right|^{2} d s \leqq L\left[\omega(1 / n) / n^{k}\right]^{2} .
$$

It is known [8, pp. 345-346] that Cauchy's integral formula is valid for $f(z)$ where the integral is taken along $C_{R}$. Choose $\pi_{n}(z)$ as the polynomial in $z$ of 
degree $n$ which interpolates to $f(z)$ in $n+1$ equally-distributed points on $C$. (The " $n+1$ equally-distributed points" are the images in the $z$-plane of the $(n+1)$ st roots of unity under the conformal map of $|w|>1$ onto the exterior of $C$ by $z=\psi(w)$.) Then Theorem 7.1 follows from (7.2) and known results $[10$, Theorems $3.3,4.7]$.

Corresponding direct theorems for Problem $\beta$ when approximation is measured by line and surface integrals are trivial corollaries of Theorem 7.1; their statement is left to the reader.

8. Problem $\beta$, indirect. Tchebycheff theorems. Let $C$ be a rectifiable Jordan curve; suppose $z_{0}$ is a point interior to $C$. Let $g\left(z_{0}, z\right)$ denote Green's function for the interior of $C$ with pole at $z_{0}$, and let $\nu$ denote the inner normal; $g\left(z_{0}, z\right)$ is chosen to become positively infinite at $z_{0}$. With this notation we make

Definition 8.1. A curve $C$ is said to be of Type B if $C$ is a rectifiable Jordan curve such that for some $q>0$ the integral $\int_{C}\left[\partial g\left(z_{0}, z\right) / \partial \nu\right]^{-q} d s$ exists.

We prove now

Theorem 8.1. Let $C$ be a curve of Type B. Let $u(z)$ be defined on $\bar{C}$ and suppose there exist harmonic polynomials $p_{n}(z)$ such that

$$
\left|u(z)-p_{n}(z)\right| \leqq \frac{\Omega(n)}{n^{k+1} R^{n}}, \quad \quad \text { o on } \bar{C}, R>1 .
$$

Set $f(z)=u(z)+i v(z)$, where $v(z)$ is conjugate to $u(z)$. Then $f(z)$ is analytic in the interior of $C_{R}$ and continuous on $\bar{C}_{R} ; f^{(k)}(z)$ exists and is continuous on $\bar{C}_{R}$ with modulus of continuity which satisfies a condition of form (5.2). Hence $\partial^{k} u / \partial s^{k}$ exists and is continuous on $C_{R}$ with modulus of continuity which satisfies a condition of the same form.

Here and in Theorem 8.2 below, if $u(z)$ and $f(z)$ are not originally assumed to be defined on the entire point set considered, the supplementary definition is to be made by means of the convergent series of polynomials.

Let $z_{0}$ be a point inside $C$. Define $\pi_{n}(z)=p_{n}(z)+i q_{n}(z), q_{n}\left(z_{0}\right)=v\left(z_{0}\right)$. It follows from (8.1) that

$$
\left|p_{n+1}(z)-p_{n}(z)\right| \leqq M_{1} \Omega(n) / n^{k+1} R^{n}, \quad z \text { on } \bar{C} .
$$

Hence by a known result [11, Theorem 9.6] we have

$$
\left|\pi_{n+1}(z)-\pi_{n}(z)\right| \leqq M_{2} \Omega(n) / n^{k+1}, \quad z \text { on } \bar{C}_{R} .
$$

By virtue of (8.1) and (8.2) we thus obtain

$$
\begin{gathered}
f(z)=\pi_{1}(z)+\left[\pi_{2}(z)-\pi_{1}(z)\right]+\left[\pi_{3}(z)-\pi_{2}(z)\right]+\cdots \\
\left|f(z)-\pi_{n}(z)\right| \leqq M_{2} \sum_{j=n}^{\infty} \Omega(j) / j^{k+1} \leqq M_{3} \Omega(n) / n^{k}, \quad z \text { on } \bar{C}_{R} .
\end{gathered}
$$


Theorem 8.1 now follows from (8.3) and Theorems 5.6 and 2.7.

For the analytic case a similar theorem can be established under a weaker hypothesis on $C$ by employing a well known result $[9$, pp. 77-78] together with the method used to prove Theorem 8.1:

THEOREM 8.2. Let $E$ with boundary $C$ be a closed limited point set whose complement is connected and regular; let $C_{R}, R>1$, be a contour. Let $f(z)$ be defined on $E$, and suppose there exist polynomials $\pi_{n}(z)$ in $z$ such that

$$
\left|f(z)-\pi_{n}(z)\right| \leqq \Omega(n) / n^{k+1} R^{n},
$$

z on $E$.

Then the conclusion of Theorem 8.1 is valid.

When we say that the complement $E^{*}$ of $E$ is "regular" we mean that $E^{*}$ possesses a Green's function with pole at infinity.

Walsh and Sewell [10] have established Theorem 8.2 by the same method for the special case $\Omega(n)=M_{1} n^{-\alpha}$.

9. Problem $\beta$, indirect. Integral measures of approximation. To prove indirect theorems for Problem $\beta$ where approximation is measured by a line integral, we shall need

Theorem 9.1. Let $C$ satisfy the hypothesis of Theorem 4.1 ; let $z_{0}$ be a point inside C. Let $\pi_{n}(z)=p_{n}(z)+i q_{n}(z), q_{n}\left(z_{0}\right)=0$, be a polynomial in $z$. If $p>1$, then

$$
\left|\pi_{n}(z)\right| \leqq L R^{n}\left[\int_{C}\left|p_{n}(z)\right| p d s\right]^{1 / p}, \quad \text { zon } \bar{C}_{R}, R>1
$$

Theorem 4.1 and the hypothesis yield

$$
\int_{C}\left|\pi_{n}(z)\right|^{p} d s \leqq M \int_{C}\left|p_{n}(z)\right|^{p} d s .
$$

Theorem 9.1 now follows from a result due to Walsh [9, pp. 92-93].

As an indirect theorem for approximation measured by a line integral we prove

THEOREM 9.2. Let $C$ satisfy the hypothesis of Theorem 4.1. Let $U(z)$ be defined on $C$, and suppose there exist harmonic polynomials $p_{n}(z)$ such that

$$
\int_{C} \rho(s)\left|U(z)-p_{n}(z)\right|^{p} d s \leqq\left[\frac{\Omega(n)}{n^{k+1} R^{n}}\right]^{p}, \quad p>1, R>1,
$$

where $\rho(s)$ is positive, integrable, and bounded from zero. Then $\lim _{n \rightarrow \infty} p_{n}(z)$, $z$ in $\bar{C}_{R}$, exists. If $u(z)=\lim _{n \rightarrow \infty} p_{n}(z), z$ in $\bar{C}_{R}$, then $U(z)=u(z)$ almost everywhere on $C$ and the conclusion of Theorem 8.1 is valid for $u(z)$ and $f(z)$.

From (9.1) we obtain by the use of (4.1) 


$$
\int_{C}\left|p_{n+1}(z)-p_{n}(z)\right|^{p} d s \leqq M\left[\Omega(n) / n^{k+1} R^{n}\right]^{p} .
$$

Choose $r, 1<r<R$; then by Theorem 9.1

$$
\left|p_{n+1}(z)-p_{n}(z)\right| \leqq M_{1} r^{n} \Omega(n) / n^{k+1} R^{n}, \quad z \text { on } \bar{C}_{r} .
$$

On expanding $u(z)$ in the usual way we have for $z$ in $\bar{C}_{r}$ :

$$
\left|u(z)-p_{n}(z)\right| \leqq M_{1} \sum_{\mu=n}^{\infty}{ }^{\mu} \Omega(\mu) / \mu^{k+1} R^{\mu} \leqq M_{2} \Omega(n) / n^{k+1}(R / r)^{n} .
$$

Theorem 9.2 follows from Theorem 8.1 if we take as the curve $C$ of that theorem the present $C_{r}$ and as $R$ of that theorem the present $R / r$.

The method used to establish Theorem 9.2 and a result due to Walsh [9, pp. 92-93] enable us to prove

Theorem 9.3. Let $C$ be a rectifiable Jordan curve; let $\rho(s)$ be positive, integrable, and bounded from zero. Let $F(z)$ be defined on $C$; suppose polynomials $\pi_{n}(z)$ in $z$ exist such that

$$
\int_{C} \rho(s)\left|f(z)-\pi_{n}(z)\right|^{p} d s \leqq\left[\Omega(n) / n^{k+1} R^{n}\right]^{p}, \quad p>0, R>1 .
$$

Then $\lim _{n \rightarrow \infty} \pi_{n}(z), z$ in $\bar{C}_{R}$, exists. If $f(z)=\lim _{n \rightarrow \infty} \pi_{n}(z), z$ in $\bar{C}_{R}$, then $F(z)=f(z)$ almost everywhere on $C$ and the conclusion of Theorem 8.1 is valid for $f(z)$ and $u(z)$.

We turn now to indirect theorems for Problem $\beta$ when degree of approximation is measured by a surface integral. We must first establish certain results on polynomials. Theorems 9.4-9.6 which follow are valid for any polynomial $\pi_{n}(z)$ in $z$ of degree $n$. Western [12, pp. 861-863] has proved:

THEOREM 9.4. If $C$ is an analytic Jordan curve, then

$$
\iint_{\bar{c}}\left|\pi_{n}^{\prime}(z)\right|^{p} d A \leqq M n^{p} \iint_{\bar{C}}\left|\pi_{n}(z)\right|^{p} d A, \quad p>1 .
$$

To be sure, Western states that the theorem is true for $n$ sufficiently large; however, if the constant $M$ is chosen suitably, the theorem will clearly hold for all positive integers $n$, since a polynomial of degree $n$ is also a polynomial of degree $m \geqq n$.

Western's result enables us to prove

THEOREM 9.5. Let $C$ be an analytic Jordan curve; then

$$
\int_{C}\left|\pi_{n}(z)\right|^{p} d s \leqq \operatorname{Ln} \iint_{\bar{c}}\left|\pi_{n}(z)\right|^{p} d A, \quad \quad p>1 .
$$


Let $z=\chi(w)$ map $|w|<1$ one-to-one and conformally onto the interior of $C$. Set $F_{n}(w)=w^{2} \pi_{n}[\chi(w)], w=r e^{i \theta}$. Suppose $\theta$ is such that $F_{n}\left(r e^{i \theta}\right) \neq 0$, $0<r \leqq 1$. Then for every $\epsilon, 0<\epsilon<1$, we have

$$
\left[F_{n}\left(e^{i \theta}\right)\right]^{p}-\left[F_{n}\left(\epsilon e^{i \theta}\right)\right]^{p}=\int_{\epsilon e^{i \theta}}^{e^{i \theta}} \frac{d}{d w}\left(\left[F_{n}(w)\right]^{p}\right) d w,
$$

where we choose a particular branch of $\left[F_{n}(w)\right]^{p}$ and take the integral along the straight line joining $\epsilon e^{i \theta}$ and $e^{i \theta}$. It follows that

$$
\left|F_{n}\left(e^{i \theta}\right)\right|^{p} \leqq\left|F_{n}\left(\epsilon e^{i \theta}\right)\right|^{p}+p \int_{\epsilon}^{1}\left|F_{n}\left(r e^{i \theta}\right)\right|^{p-1}\left|F_{n}^{\prime}\left(r e^{i \theta}\right)\right| d r .
$$

Let $\epsilon \rightarrow 0$. Since both terms of the second member of (9.2) are continuous, single-valued functions of $\epsilon, 0 \leqq \epsilon<1$, and $F_{n}(0)=0$, we obtain for all such $\theta$ the relation

$$
\left|F_{n}\left(e^{i \theta}\right)\right|^{p} \leqq p \int_{0}^{1}\left|F_{n}\left(r e^{i \theta}\right)\right|^{p-1}\left|F_{n}^{\prime}\left(r e^{i \theta}\right)\right| d r .
$$

Now let $\theta_{0}$ be such that $F_{n}\left(r_{0} e^{i \theta_{0}}\right)=0$ for some $r_{0}, 0<r_{0} \leqq 1$. Since $F_{n}(w)$ has at most $n+2$ zeros in $|w| \leqq 1$, there exists an $\eta>0$ such that $F_{n}\left(r e^{i \theta}\right) \neq 0$ for $\theta_{0}-\eta \leqq \theta<\theta_{0}, 0<r \leqq 1$. Therefore inequality (9.3) is valid for all $\theta, \theta_{0}-\eta$ $\leqq \theta<\theta_{0}$. On allowing $\theta$ to approach $\theta_{0}$ we obtain (9.3) for $\theta=\theta_{0}$; thus (9.3) is valid for all $\theta$. Hence

$$
\begin{aligned}
\int_{C}\left|\pi_{n}(z)\right|^{p} d s & \leqq M \int_{0}^{2 \pi}\left|F_{n}\left(e^{i \theta}\right)\right|^{p} d \theta \\
& \leqq p M \int_{0}^{2 \pi} d \theta \int_{0}^{1}\left|F_{n}\left(r e^{i \theta}\right)\right|^{p-1}\left|F_{n}^{\prime}\left(r e^{i \theta}\right)\right| d r \\
& \leqq p M \iint_{|w| \leqq 1}\left|\pi_{n}[\chi(w)]\right|^{p-1} \mid 2 \pi_{n}[\chi(w)] \\
& \left.\leqq M_{1} \iint_{\bar{c}}\left|\pi_{n}(z)\right|^{p} d A+M_{2} \iint_{\bar{c}}\left|\pi_{n}^{\prime}(z)\right| \chi(w)\right]\left.\right|^{p-1}\left|\pi_{n}^{\prime}(z)\right| d A \\
& \leqq M_{1} \iint_{\bar{c}}\left|\pi_{n}(z)\right|^{p} d A+M_{2}\left[\iint_{\bar{c}}\left|\pi_{n}(z)\right|^{p} d A\right]^{1-1 / p} \\
& \cdot\left[\iint_{\bar{c}}\left|\pi_{n}^{\prime}(z)\right|^{p} d A\right]^{1 / p} \cdot
\end{aligned}
$$

Application of Theorem 9.4 yields the desired inequality. 
We are now in a position to prove

Theorem 9.6. If $C$ is an analytic Jordan curve and $p>1$, then

$$
\left|\pi_{n}(z)\right| \leqq L n^{1 / p} R^{n}\left[\iint_{\bar{C}}\left|\pi_{n}(z)\right|^{p} d A\right]^{1 / p}, \quad z \text { on } \bar{C}_{R}, R>1 .
$$

Let $w=\phi(z)$ map the exterior of $C$ one-to-one and conformally onto the exterior of $|w|=1$ so that the points at infinity in the two planes correspond to each other. Then

$$
\frac{\pi_{n}(z)}{[\phi(z)]^{n+1}}=\frac{1}{2 \pi i} \int_{C} \frac{\pi_{n}(t)}{[\phi(t)]^{n+1}(t-z)} d t, \quad z \text { on } C_{R}, R>1 .
$$

Hence using Theorem 9.5 we obtain

$$
\begin{aligned}
\left|\pi_{n}(z)\right| & \leqq M R^{n} \int_{C}\left|\pi_{n}(t)\right||d t| \leqq M_{1} R^{n}\left[\int_{C}\left|\pi_{n}(t)\right| p|d t|\right]^{1 / p} \\
& \leqq M_{2} n^{1 / p} R^{n}\left[\iint_{\bar{C}}\left|\pi_{n}(t)\right|^{p} d A\right]^{1 / p}, \quad z \text { on } \bar{C}_{R} .
\end{aligned}
$$

As a consequence of Theorems 4.1 and 9.6 we have

Theorem 9.7. Let $C$ be an analytic Jordan curve; let $z_{0}$ be a point inside $C$. Let $\pi_{n}(z)=p_{n}(z)+i q_{n}(z), q_{n}\left(z_{0}\right)=0$, be a polynomial in $z$. If $p>1$, then

$$
\left|\pi_{n}(z)\right| \leqq L n^{1 / p} R^{n}\left[\iint_{\bar{C}}\left|p_{n}(z)\right|^{p} d A\right]^{1 / p}, \quad z \text { on } \bar{C}_{R}, R>1 .
$$

The method used in proving Theorem 9.2 together with Theorem 9.7 yields

THEOREM 9.8. Let $C$ be an analytic Jordan curve; let $\rho(z)$ be positive, integrable, and bounded from zero on $\bar{C}$. Let $U(z)$ be defined on $\bar{C}$ and suppose there exist harmonic polynomials $p_{n}(z)$ such that

$$
\iint_{\bar{c}} \rho(z)\left|U(z)-p_{n}(z)\right|^{p} d A \leqq \frac{[\Omega(n)]^{p}}{n^{k p+p+1} R^{n p}}, \quad p>1, R>1 .
$$

Then $\lim _{n \rightarrow \infty} p_{n}(z), z$ in $\bar{C}_{R}$, exists. If $u(z)=\lim _{n \rightarrow \infty} p_{n}(z), z$ in $\bar{C}_{R}$, then $U(z)$ $=u(z)$ almost everywhere on $\bar{C}$, and the conclusion of Theorem 8.1 is valid.

\section{BIBLIOGRAPHY}

1. N. Achyèser, Über den Jacksonschen Approximationssatz, Communications de la Société Mathématique de Kharkow (4) vol. 8 (1934) pp. 3-12.

2. John Curtiss, $A$ note on the degree of polynomial approximation, Bull. Amer. Math. Soc. vol. 42 (1936) pp. 873-878. 
3. C. de la Vallée Poussin, Sur l'intégrale de Lebesgue, Trans. Amer. Math. Soc. vol. 16 (1915) pp. 435-501.

4. - Lȩ̧ons sur l'approximation des fonctions d'une variable réelle, Paris, 1919.

5. J. Privaloff, Sur certaines propriétés métriques des fonctions analytiques, J. École Polytech. (2) vol. 24 (1924) pp. 77-112.

6. M. Riesz, Sur les fonctions conjuguées, Math. Zeit. vol. 27 (1928) pp. 218-244.

7. W. E. Sewell, Degree of approximation by polynomials in the complex domain, Annals of Mathematics Studies, no. 9, Princeton, 1942.

8. V. Smirnoff, Sur les formules de Cauchy et de Green, Bull. Acad. Sci. URSS Sér. Math. (7) vol. 3 (1932) pp. 337-371.

9. J. L. Walsh, Interpolation and approximation by rational functions in the complex domain, Amer. Math. Soc. Colloquium Publications, vol. 20, New York, 1935.

10. J. L. Walsh and W. E. Sewell, Sufficient conditions for various degrees of approximation by polynomials, Duke Math. J. vol. 6 (1940) pp. 658-705.

11. J. L. Walsh, W. E. Sewell, and H. M. Elliott, On the degree of polynomial approximation to harmonic and analytic functions, Trans. Amer. Math. Soc. vol. 67 (1949) pp. 381-420.

12. D. W. Western, Inequalities of the Markoff and Bernstein type for integral norms, Duke Math. J. vol. 15 (1948) pp. 839-869.

WASHINGTON UNIVERSITY, ST. Louis, Mo. 